\title{
Electronic Medical Records as a Research Tool: Evaluating Topiramate Use at a Headache Center.
}

\author{
Michael J. Marmura, MD \\ Thomas Jefferson University \\ Mary Hopkins, RN, MSN \\ Thomas Jefferson University \\ Jocelyn Andrel, MSPH \\ Thomas Jefferson University \\ William B. Young, MD \\ Thomas Jefferson University \\ Pavid Ahis Bipadidefitional works at: https://jdc.jefferson.edu/neurologyfp \\ Thomas Jefferson University \\ Part of the Neurology Commons \\ $\frac{\text { Let us know how access to this document benefits you }}{\text { See next page for additional authors }}$
}

\section{Recommended Citation}

Marmura, MD, Michael J.; Hopkins, RN, MSN, Mary; Andrel, MSPH, Jocelyn; Young, MD, William B.; Biondi, DO, David M.; Rupnow, PhD, Marcia F.T.; and Armstrong, MD, Robert B., "Electronic Medical Records as a Research Tool: Evaluating Topiramate Use at a Headache Center." (2010). Department of Neurology Faculty Papers. Paper 32.

https://jdc.jefferson.edu/neurologyfp/32

This Article is brought to you for free and open access by the Jefferson Digital Commons. The Jefferson Digital Commons is a service of Thomas Jefferson University's Center for Teaching and Learning (CTL). The Commons is a showcase for Jefferson books and journals, peer-reviewed scholarly publications, unique historical collections from the University archives, and teaching tools. The Jefferson Digital Commons allows researchers and interested readers anywhere in the world to learn about and keep up to date with Jefferson scholarship. This article has been accepted for inclusion in Department of Neurology Faculty Papers by an authorized administrator of the Jefferson Digital Commons. For more information, please contact: JeffersonDigitalCommons@jefferson.edu. 


\section{Authors}

Michael J. Marmura, MD; Mary Hopkins, RN, MSN; Jocelyn Andrel, MSPH; William B. Young, MD; David M. Biondi, DO; Marcia F.T. Rupnow, PhD; and Robert B. Armstrong, MD 
Electronic Medical Records as a research tool: evaluating topiramate use at a headache center

M.J. Marmura MD, M. Hopkins RN, MSN, J. Andrel, MSPH, W.B. Young, MD, D. M. Biondi, DO, M.F.T. Rupnow, PhD*, and R. B. Armstrong, MD

From the Jefferson Headache Center (M.J.M., M.H., J.A., W.B.Y.), Philadelphia, PA; and OrthoMcNeil Janssen Scientific Affairs, LLC (D.B., M.F.T.R., R.A.), Raritan, NJ.

*Current affiliation: Ethicon, Inc., Somerville, NJ.

Address correspondence to: Michael Marmura, Thomas Jefferson University, Jefferson

Headache Center, 111 S. $11^{\text {th }}$ St. Suite 8130 Philadelphia, PA 19107

Tel: 215-955-2243; Fax: 215-955-2060; Email: Michael.Marmura@jefferson.edu 


\section{Disclosures}

Mary Hopkins and Jocelyn Andrel report no conflicts of interest. Dr. Marmura has a member of the speaker's bureau for Cephalon and received research or education grants from Merck and GlaxoSmithKline. Dr. Young has been an advisor and a member of the speaker's bureau for Allergan, GlaxoSmithKline, Merck, Ortho-McNeil Janssen, Valeant, and received research or education grants from AGA Medical, Advanced Bionics, Advanced Neuromodulation Systems, Allergan, Capnia, Eli Lilly, Endo Pharmaceuticals, GlaxoSmithKline, Medtronic, Merck, Minster, and Valeant. Dr. Rupnow was an employee of Ortho-McNeil Janssen Scientific Affairs, LLC, a Johnson \& Johnson company, at the time of manuscript development. Dr. Biondi and Dr. Armstrong are employees of Ortho-McNeil Janssen Scientific Affairs, LLC, a Johnson \& Johnson company. Jocelyn Andrel (Thomas Jefferson University) conducted the statistical analyses. 
Abstract—Background: Electronic medical records (EMRs) are used in large healthcare centers

to increase efficiency and accuracy of documentation. These databases may be utilized for clinical research or to describe clinical practices such as medication usage, Methods: We conducted a retrospective analysis of EMR data from a headache clinic to evaluate clinician prescription use and dosing patterns of topiramate. The study cohort comprised 4833 unique deidentified records, which were used to determine topiramate dose and persistence of treatment. Results: Within the cohort, migraine was the most common headache diagnosis $(\mathrm{n}=3753$,

Deleted: To date, the Deleted: utility of these Deleted: in Deleted: the use of

Deleted: has not been fully evaluated

Deleted: To explore EMR as a clinical research tool and determine the use of topiramate and factors related to its use, we conducted a retrospective analysis from EMR data collected at the Jefferson Headache Center in Philadelphia.

Deleted: for the

$77.7 \%)$, followed by tension-type headache $(n=338,7.0 \%)$ and cluster or trigeminal autonomic cephalalgias $(n=287,5.9 \%)$. Physicians prescribed topiramate more often for subjects with migraine and idiopathic intracranial hypertension $(\mathrm{IIH})(p<0.0001)$ than for those with other conditions, and more often for subjects with coexisting conditions including obesity, bipolar disorder, and depression. The most common maintenance dose of topiramate was $100 \mathrm{mg} /$ day; however, approximately $15 \%$ of subjects received either less than $100 \mathrm{mg} /$ day or more than 200 $\mathrm{mg}$ /day. More than a third of subjects were prescribed topiramate for more than 1 year, and subjects with a diagnosis of migraine were prescribed topiramate for a longer period of time than those without migraine. Conclusions: Findings from our study using EMR demonstrate that physicians use topiramate at many different doses and for many off-label indications. This analysis provided important insight into our patient populations and treatment patterns. 
Introduction. Electronic Medical Records (EMRs) have the potential to improve clinical efficiency and documentation. Recently, health care providers have begun using EMRs for clinical research. Most large-scale retrospective observational studies conducted to date have been based on insurance claims data (e.g., prescriptions filled to determine commonly prescribed doses of a medication). ${ }^{1}$ EMRs vary widely in their structure, capacity, and extent of data capture. Analysis is most successful in evaluating objective data, such as lab results. Subjective or complex variables can be more challenging to measure and document. Results from clinical practice may vary tremendously compared with the results of clinical trials. EMRs do not solve all issues with documentation; they can help standardize documentation but do not prevent inaccuracies. ${ }^{2}$ Most EMRs in clinical practice are designed to increase the accuracy of billing, eliminate the need for dictation, improve communication of health information between clinicians, and prevent errors rather than for use as a research tool.

Retrospective EMR analyses have yielded important findings that can affect clinical care, such as studies showing increased cardiovascular event rates with the use of rofecoxib. ${ }^{3}$ Other EMR studies have documented prevention of medication errors ${ }^{4}$ and demonstrated trends in treatment response that may lead to better practice. For instance, a recent EMR analysis demonstrated that antiepileptic usage in elderly patients has not changed significantly despite changes in clinical guidelines. ${ }^{5}$ EMR studies can also help determine compliance with treatment guidelines and demonstrate cost savings. ${ }^{6}$ The goal of this pilot study was to explore the utility of EMR as a clinical research tool through an evaluation of patient demographics, diagnoses, and topiramate use in a cohort of patients treated at a university-based headache specialty clinic. 
Methods. This study was a retrospective analysis of de-identified, aggregate EMR data from the Jefferson Headache Center in Philadelphia, Pennsylvania. A waiver of authorization for use of personal health information and Institutional Review Board approval was obtained.

The Jefferson Headache Center utilizes Centricity® Physician Office (formerly Logician) to document and maintain patients' medical records. All unique records with an initial office visit from April 1, 2000 through June 30, 2006 were evaluated and categorized into two patient groups: those who had received at least one prescription order for topiramate during the study period, and those who had not. The no topiramate group included patients who were never prescribed topiramate, or whose prescription start and stop date occurred before the study period, had an order entered and removed the same day, or had only a single order with instructions to taper off topiramate. All records have a clinical date-time stamp that is independent of the physician or nurse user. There are no required fields; the EMR system and documentation are driven by clinical care. The patient identifiers are system-generated markers that maintain related records. Medical diagnoses were recoded using both the ICD-9-CM code and description.

Conditions that might influence topiramate use were examined, including diabetes, hypertension, obesity, epilepsy, anxiety, depression, bipolar disorder, tremor, and fibromyalgia.

To ascertain dose and length of time on topiramate, tables containing the medication description (product name, dose form, strength) and instructions for each patient were merged with tables of prescription quantities, refills, and associated medication order key. The type of prescription, date, and time that the prescription was written were used to remove duplicates, and all prescription reprints were deleted. When there were multiple prescriptions for the same order on the same date with different pill quantities, the latest timed orders were retained. Maintenance dose was defined as the daily dose ordered for the longest total period of time. The start date for 
determining total persistence of treatment was the first order date. If a patient had a documented stop date, it was used as the end date, and no further calculation was done. In instances where a patient did not have a documented stop date, the end date was calculated based on the last prescription date plus the pill supply days. We assumed the medication was taken as directed.

Statistical methods. Analyses were conducted on the entire study group (patients with and patients without a topiramate prescription order) and then separately within the group of patients with topiramate prescription orders. Descriptive statistics such as means, medians, and frequencies between groups were calculated for age, race, gender, marital status, diagnoses, number of office visits, and contact time. Significance was tested using the Pearson chi-square test for categorical outcomes; the t-test or Wilcoxon two-sample test were used for continuous outcomes. Variables with a time dimension, such as persistence on topiramate, were analyzed separately by year. Within the group of patients with topiramate prescription orders, medication usage was similarly explored based on diagnoses, such as migraine or cluster headache. Simple logistic regression was used to determine any associations between patient factors (such as diagnosis) and topiramate use. Significance was assessed using the Wald Chi-squared statistic. No adjustments for multiplicity were made due to the exploratory nature of this study. Statistical analyses were done using SAS 9.1 (SAS Institute, Cary, NC).

Results. We extracted 4833 unique records with an initial visit during the study period of April 1, 2000 through June 30, 2006. Study demographics are summarized in Table 1; most patients were white, female, and married at the time of the study. Race, gender, and age were all significantly associated with a topiramate prescription order but these results were complicated by missing data. Race was a missing field for 376 patients and 122 had no listed martial status. 
Subjects with a topiramate order were on average 2.65 years younger than nonusers (mean age \pm SD: $39.30 \pm 12.63$ vs $41.95 \pm 15.99, p<0.001)$.

The median number of clinic visits was six, and the mean visit number per record was eight. Patients who received prescription orders for topiramate had a greater number of clinic visits, with a median of nine, compared with a median of three visits for those without a topiramate order $(p<0.001)$. Patients who received topiramate continued their care at the Jefferson Headache Center for longer than those who did not (median: 378.5 days vs 63 days; $p<0.001$ ).

Diagnoses. Patients evaluated at the Jefferson Headache Center were found to have diagnoses in 22 different categories. Primary headaches were most common, and migraine was the most common headache type: migraine $(n=3753 ; 77.7 \%)$, tension-type headache $(n=338 ; 7.0 \%)$, and cluster or trigeminal autonomic cephalalgias $(n=287,5.9 \%)$. Secondary headache diagnoses, either alone or in addition to a primary headache diagnosis, were common $(\mathrm{n}=1413$; $29.2 \%$ ) and included cervicalgia, post-traumatic headache, idiopathic intracranial hypertension (IIH), tumor, cerebrovascular accident, or aneurysm. A total of $2443(50.5 \%)$ patients had multiple headache diagnoses. The most common diagnosis combinations were: chronic migraine and medication overuse headache $(\mathrm{n}=558,11.5 \%)$, migraine without aura and chronic migraine $(\mathrm{n}=512,10.6 \%)$, migraine with and without aura $(\mathrm{n}=304,6.3 \%)$, and migraine with aura and chronic migraine $(n=285,5.9 \%)$.

At the Jefferson Headache Clinic, most prescription orders for topiramate were for patients with a diagnosis of migraine. Physicians ordered topiramate more often for patients with a diagnosis of migraine or IIH and less often for patients with tension headache, cluster headache, or cranial neuralgias such as trigeminal neuralgia. Patients who were overweight or obese, had bipolar 
disorder, depression, seizures, tremors, or fibromyalgia received a prescription order for topiramate more frequently than the rest of the patient cohort (Table 2).

Dosing. Topiramate was prescribed at doses ranging from as low as $15 \mathrm{mg}$ every other day to as high as $1600 \mathrm{mg}$ per day. Figure 1 shows the distribution of 2192 patients who received a dose of topiramate, by the maintenance and maximum daily topiramate dose. Maintenance dose was defined as the dose prescribed for the longest period of time during the study. The most frequently prescribed maintenance dose was $100 \mathrm{mg} /$ day $(\mathrm{n}=750,34.2 \%)$. Our findings also revealed that $329(15.0 \%)$ patients were prescribed a maintenance dose less than $100 \mathrm{mg} / \mathrm{day}$ and $332(15.1 \%)$ patients were prescribed a maintenance dose greater than $200 \mathrm{mg}$ per day. The median topiramate daily dose $(125 \mathrm{mg})$ was the same in migraine and non-migraine patients. The median topiramate daily dose (100 mg) was not significantly different in patients with tensiontype headache than patients without a diagnosis of tension-type headache (125mg). IIH was the only headache diagnosis for which the median maintenance daily dose was higher than those with all other headache diagnoses. (200 mg vs $125 \mathrm{mg} ; p=0.0106)$.

Many factors can influence the dosing of topiramate, including the headache diagnosis and coexisting medical conditions. Patients with a diagnosis of migraine, particularly chronic migraine, were significantly more likely to have a diagnosis of depression. Of the 3753 patients in the "All migraine" category, 1241 (33\%) also had a diagnosis of depression (odds ratio [OR], $1.21 ; 95 \%$ confidence interval $[\mathrm{CI}], 1.04$ to $1.40 ; p=0.0114)$, and of the 2265 patients in the "chronic migraine" category, 945 (41.7\%) also had a diagnosis of depression (OR, 2.30; 95\% CI, 2.04 to $2.61 ; p=<0.001$ ). Patients with depression taking topiramate (830 of 1554 patients, $53.4 \%)$ received a higher median daily dose than patients without a diagnosis of depression (150 mg vs $100 \mathrm{mg} ; p=0.0272)$. On the other hand, patients with anxiety disorders who were 
prescribed topiramate (505 of 1053 patients, 48.0\%) received a lower median topiramate dose than patients without the diagnosis $(100 \mathrm{mg}$ vs $150 \mathrm{mg} ; p=0.0372)$. One hundred and fifteen of $158(72.8 \%)$ patients with an overweight or obesity diagnosis were prescribed topiramate and received a higher median daily dose of topiramate compared with patients without a diagnosis of being overweight or obese, but this was not significant (150 mg vs $125 \mathrm{mg} ; p=0.1583$ ).

Persistence of treatment. Persistence of topiramate treatment was similar whether computed by the period patients took topiramate, based on prescription order data or by projection from the amount of pills taken. Figure 2 shows the proportion of patients and the total time that patients took topiramate. More than one third of all patients took topiramate for more than 1 year. Of the 2192 patients who received topiramate during the study period, 2098 had at least two orders or one order and a prescription, allowing a dimension of persistence on the drug to be calculated. Six hundred and eighty three of the 2098 (32.6\%) patients had topiramate on their current medication list and had a prescription in the final 6 months of the study. In all years during the study period, most patients who received topiramate, had a diagnosis of migraine. During the entire study period (2000-2006), patients with migraine remained on topiramate an average of 108 days longer than patients without migraine. Persistence was longer for patients with migraine who began therapy in the years $2001,2003,2004$, and $2005(p=0.0349,0.0192$, 0.0346, and 0.0144), and marginally so for patients who began taking topiramate in 2002 ( $p=$ 0.0781), compared with patients without a diagnosis of migraine (table 3 ).

Discussion. This study provides insight into the usefulness of EMR as a clinical research tool through an assessment of how practitioners at a university referral center used topiramate in everyday clinical practice for the treatment of patients with headache. Results indicate that clinicians at the headache center prescribe topiramate to treat many different types of headaches. 
Topiramate is approved by the United States Food and Drug Administration (FDA) for the prevention of migraine in adults, so it is not surprising that physicians at the headache center had prescribed topiramate for nearly half of the patients in the study population. Patients who were younger, female, and white were more likely to have received topiramate, probably because these patients were more likely to have a diagnosis of migraine and chronic migraine than those in the no topiramate group. Providers were more likely to prescribe topiramate during treatment for patients with more clinic visits, possibly because the headache center's physicians tend to prescribe topiramate more often for cases of refractory headache. Our EMR does not require physicians to distinguish between chronic and episodic migraine, and many patients have both a chronic and episodic diagnosis, as patients often improve or worsen with treatment. This makes it difficult to determine if different doses are needed for those with chronic migraine.

In our study, topiramate was prescribed for uses that have not been approved by the FDA, such as IIH. Recent studies have shown topiramate to be effective for IIH. ${ }^{12,13}$ Many patients with secondary headaches, cluster headache, and cranial neuralgias also received topiramate. Some small studies have shown that topiramate can be helpful for patients with trigeminal neuralgia ${ }^{14,15}$ or cluster headache. ${ }^{16}$ Our study also indicates that topiramate was more likely to have been prescribed for patients with coexisting medical diagnoses such as depression, bipolar disorder, and obesity. This finding could be related to the fact that depression and bipolar disorder are co-morbid conditions in chronic migraine; while obesity has been implicated as a risk factor for developing chronic migraine. ${ }^{17}$ Physicians may also have prescribed topiramate with the hope of inducing weight loss in obese patients.

The variability of the daily doses of topiramate in the study was notable. Physicians prescribed, on average, higher doses of topiramate for patients with chronic migraine or IIH. Clinical studies 
to date have used daily doses of $200 \mathrm{mg}$ or less for migraine prevention. In a study of patients with episodic migraine, topiramate $200 \mathrm{mg} /$ day was no more effective than $100 \mathrm{mg} / \mathrm{day} .{ }^{18}$ In contrast, a minority of patients seen at the Jefferson Headache Clinic received doses higher than $200 \mathrm{mg} / \mathrm{day}$, perhaps because they metabolized the medication more efficiently, were more resistant to medication-related side effects, and/or had conditions that were less responsive to usual treatment. Other patients received daily doses less than $100 \mathrm{mg}$, often for extended periods of time. Paresthesias, a common topiramate side effect, may be bothersome to anxious patients, and may explain why they tend to be on lower doses. The study emphasizes the importance of individualizing treatment.

$\underline{\text { Headache classification and practice evolved over the specified study period, leading to an }}$ increasing tendency for physicians to enter more than one headache diagnosis into the EMR. For example, the diagnosis of transformed migraine was initially cited on the headache center's custom list as "transformed migraine with and without rebound", a combination of two distinct diagnoses. The diagnoses were eventually separated and, for the "with rebound" diagnosis segment, the nomenclature was changed to medication overuse headache to align with new diagnostic terminology. Moreover, many patients with a diagnosis of migraine received additional diagnoses, such as cervicalgia, tension headache, or cervical dystonia, to reflect these $\underline{\text { coexisting conditions and their possible contribution to the clinical presentation of the patient's }}$ $\underline{\text { migraine disorder. This practice increased as billing documentation requirements became more }}$ rigorous. One issue related to this change in documentation is that multiple diagnoses make it difficult to determine the primary reason which motivated the patient to seek treatment; EMR $\underline{\text { does not require clinicians to list the most significant illness first. }}$ 
Using EMR data for the various clinical analyses provided useful information regarding patient characteristics, diagnoses, coexisting medical conditions, and treatment patterns, but organizing and analyzing these data presented numerous challenges. Limitations in interpreting the results include the fact that most EMR systems are primarily used for billing and clinical practice documentation rather than research purposes. EMRs used for clinical practice purposes do not require the same degree of monitoring and visit-to-visit consistency that is usually required in

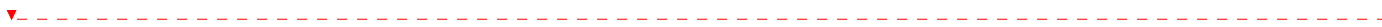
traditionally designed clinical trials. The EMR analyzed in this study had no required fields, and important data fields basic to research, such as race or marital status, often had missing data. In clinical practice, an EMR with required fields places an added time burden on clinicians, but this

lack of required data makes research more difficult. Although some patients follow up at regular intervals, others may visit the clinic more often when they are not doing well and only sporadically when they are feeling better. In addition, physicians and healthcare systems tend to vary in their documentation styles. For instance, because most patients at the Jefferson Headache Clinic are required to see a mental health provider, better availability of psychiatric diagnoses in the EMR would be expected. Many medical diagnoses were underrepresented because of the Deleted: , such as obesity, could be specialty focus of the clinicians at the headache center. Although patients fill out a questionnaire before their initial visit with a past medical history, a diagnosis is only listed in the EMR if a nurse or physician enters the diagnosis. The EMR does not automatically generate diagnoses based on data such as height and weight, so obesity in this cohort was greatly underdiagnosed due to fact that many providers did not enter the diagnosis in the EMR. Similarly, we believe that fibromyalgia, a co-morbid condition reportedly found in more than $20 \%$ of female patients with migraine, was likely underdiagnosed. ${ }^{19}$ 
The data in fields for medication and dosing were also often incomplete or difficult to analyze. For example, a prescription might have had unclear directions for administration, such as "taper as directed," requiring assumptions to be made about the intended dose. Moreover, physicians may not have documented the dispensing of drug samples to patients, perhaps affecting the initial dose and persistence analyses. In addition, patients received multiple prescriptions at the same visit. For instance, a physician would prescribe a 1-month supply of medication with three refills, but the patient would subsequently request a 90-day prescription for mail order drug delivery, which led to entry of new prescription data into the EMR. Some repeat EMR order entries were obvious, but others required manual review of the EMR to determine the actual intended dose. Finally, we needed to make assumptions about patients who lapsed in their clinic treatment. Clinic policy is not to refill prescriptions without a visit within the year.

Discontinuation was assumed, but manual review revealed that some patients who stopped taking topiramate did so because their headaches improved, they were planning to become pregnant, or they did not make a clinic visit for an extended period. Some patients continued to receive topiramate from an outside provider, and others stopped the medication only to resume it later. Additionally, the study end date placed a false stop time on those who received additional prescriptions.

Another assumption was that patients took their medication as prescribed, but many patients fail to take prophylactic medication for adequate lengths of time. Based on data from pharmacy claims, more than half of patients discontinue migraine prophylactic treatment by two months. The rate of topiramate treatment persistence at 2 months in one study was $46.4 \%$, and the treatment persistence of other common preventatives such as amitriptyline $(34.1 \%)$ and divalproex sodium $(42.7 \%)$ were even lower. ${ }^{20}$
Deleted: Headache classification and practice evolved over the specified study period, leading to an increasing tendency for physicians to enter more than one headache diagnosis into the EMR. For example, the diagnosis of transformed migraine was initially cited on the headache center's custom list as

"transformed migraine with and without rebound", a combination of two distinct diagnoses. The diagnoses were eventually separated and, for the "with rebound" diagnosis segment, the nomenclature was changed to medication overuse headache to align with new diagnostic terminology. Moreover, many patients with a diagnosis of migraine received additional diagnoses, such as cervicalgia, tension headache, or cervical dystonia, to reflect these coexisting conditions and their possible contribution to the clinical presentation of the patient's migraine disorder. This practice increased as billing documentation requirements became more rigorous. One issue related to this change in documentation is that multiple diagnoses make it difficult to determine the primary reason which motivated the patient to seek treatment; EMR does not require clinicians to list the most significant illness first.II The use of an EMR for clinical care does not necessarily facilitate clinical research The EMR analyzed in this study had no required fields, and important data fields basic to research, such as race or other patient characteristics, often had missing data. 
This pilot study extracted and analyzed data from the EMR system used at the Jefferson Headache Center. The consistency of staff and practice patterns within the Headache Center is believed to have strengthened this study's findings. Although the results can be useful for examining practice patterns and treatment trends at the Jefferson Headache Center, they cannot be generalized to all medical or headache specialty practices because of variability in patient populations and local standards of care. Likewise, the utility of this clinical research method and its findings cannot be generalized to all EMR systems, because of wide variability in system structure and format. This research study required funding and extensive review to validate the findings and understand the discrepancies. These are luxuries that are not available to the average office practice. Dedicated effort and resources to understand the clinical information available in EMRs may be underestimated or underreported. Overall, the utility of EMRs for research would be enhanced by the standardization of EMR system design. If standardized, EMRs could be a useful tool for evaluating patient populations, disease categories, treatment patterns, and clinical outcomes within and across healthcare systems or local geographic regions. With these insights, it might be possible to determine best practices as well as practices that require improvement and ongoing monitoring. Without standardization and forethought in the design or use of EMR systems, a number of limitations in the analysis of EMR data could have the consequence of producing clinical information and outcome assessments that are difficult to interpret and lack a reasonable level of confidence.

\section{Acknowledgments}

The authors would like to thank Abhijit Dasgupta, $\mathrm{PhD}$, for his assistance with statistical analyses. Editorial support was provided by Kakuri Omari (Phase Five Communications Inc., 
New York, NY), with funding from Ortho-McNeil Janssen Scientific Affairs, LLC. This study was sponsored by Ortho-McNeil Janssen Scientific Affairs, LLC, Titusville, NJ, USA. 


\section{References}

1. Hess G, Sanders KN, Hill J, Liu LZ. Therapeutic dose assessment of patient switching from atorvastatin to simvastatin. Am J Manag Care 2007;13(Suppl 3):S80-S85.

2. Ernst ME, Brown GL, Klepser TB, Kelly MW. Medication discrepancies in an outpatient electronic medical record. Am J Health Syst Pharm 2001;58:2072-2075.

3. Solomon DH, Avorn J, Sturmer T, Glynn RJ, Mogun H, Schneeweiss S. Cardiovascular outcomes in new users of coxibs and nonsteroidal antiinflammatory drugs: high-risk subgroups and time course of risk. Arthritis Rheum 2006;54:1378-1389.

4. Bates DW, Teich JM, Lee J, et al. The impact of computerized physician order entry on medication error prevention. J Am Med Inform Assoc 1999;6:313-221.

5. Pugh MJ, Van Cott AC, Cramer JA, Knoefel JE, Amuan ME, Tabares J, Ramsay RE, Berlowitz DR; Treatment In Geriatric Epilepsy Research (TIGER) team. Trends in antiepileptic drug prescribing for older patients with new-onset epilepsy: 20002004.Neurology. 2008 May 27;70(22 Pt 2):2171-8.

6. Wang SJ, Middleton B, Prosser LA, et al. A cost-benefit analysis of electronic medical records in primary care. Am J Med 2003;114:397-403.

7. Saigh O, Triola MM, Link RN. Brief report: failure of an electronic medical record tool to improve pain assessment documentation. J Gen Intern Med 2006;21:185-188.

8. Silberstein SD, Ben-Menachem E, Shank RP, Wiegand F. Topiramate monotherapy in epilepsy and migraine prevention. Clin Ther 2005;27:154-165.

9. Lampl C, Marecek S, May A, Bendtsen L. A prospective, open-label, long-term study of the efficacy and tolerability of topiramate in the prophylaxis of chronic tension-type headache. Cephalalgia 2006;26:1203-1208. 
10. Silberstein SD, Feliu AL, Rupnow MF, Blount AC, Boccuzzi SJ. Topiramate in migraine prophylaxis: long-term impact on resource utilization and cost. Headache 2007;47:500-510.

11. Poston S, Dickson M, Johnsrud M, et al. Topiramate prescribing patterns among Medicaid patients: diagnosis, comorbidities, and dosing. Clin Ther 2007;29:504-518.

12. Finsterer J, Földy D, Fertl E. Topiramate resolves headache from pseudotumor cerebri. J Pain Symptom Manage 2006;32:401-402.

13. Celebisoy N, Gökçay F, Sirin H, Akyürekli O. Treatment of idiopathic intracranial hypertension: topiramate vs acetazolamide, an open-label study. Acta Neurol Scand $2007 ; 116: 322-327$.

14. Domingues RB, Kuster GW, Aquino CC. Treatment of trigeminal neuralgia with low doses of topiramate. Arq Neuropsiquiatr 2007;65:792-794.

15. Gilron I, Booher SL, Rowan JS, Max MB. Topiramate in trigeminal neuralgia: a randomized, placebo-controlled multiple crossover pilot study. Clin Neuropharmacol 2001;24:109-112.

16. Leone M, Dodick D, Rigamonti A, et al. Topiramate in cluster headache prophylaxis: an open trial. Cephalalgia 2003;23:1001-1002.

17. Bigal ME, Lipton RB. Obesity is a risk factor for transformed migraine but not chronic tension-type headache. Neurology 2006;67:252-257.

18. Silberstein SD. Topiramate in migraine prevention. Headache 2005;45(Suppl 1):S57-S65.

19. Ifergane G, Buskila D, Simiseshvely N, Zeev K, Cohen H. Prevalence of fibromyalgia syndrome in migraine patients. Cephalalgia 2006;26:451-456.

20. Yaldo AZ, Wertz DA, Rupnow MFT, Quimbo RM. Persistence with migraine prophylaactic treatment and acute migraine medication utilization in the managed care setting. Clin Therap 2008;30(12):2452-2460. 
Table 1 Demographics

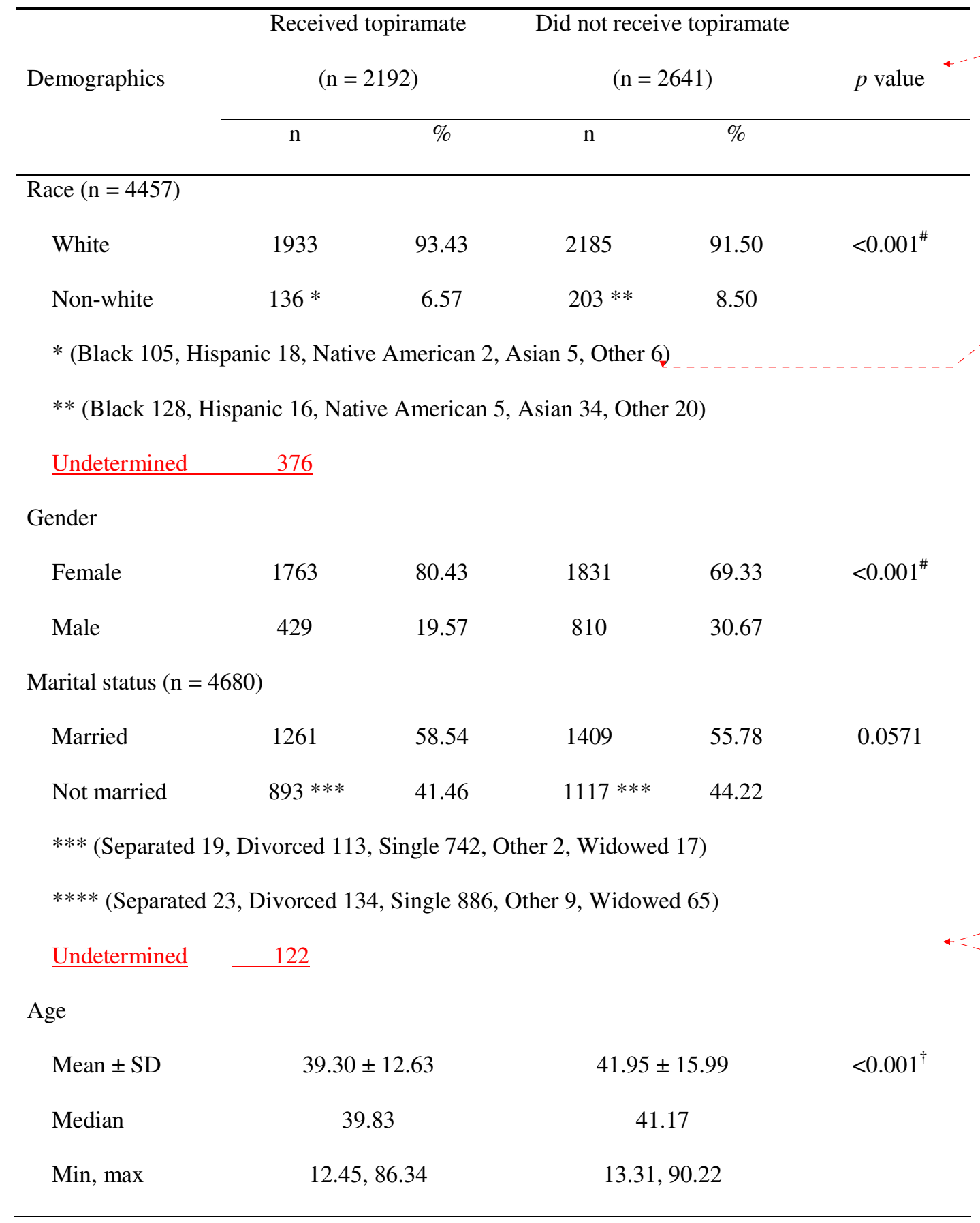

Formatted Table

Deleted: ,
Formatted: Indent: Left: 0.18 "

Formatted: Left 
${ }^{\#} p$ values derived by the $\chi^{2}$ test.

${ }^{\dagger} p$ value derived by the t-test. 
Table 2 Association of topiramate prescription by broad headache category and co-existing diagnoses

\begin{tabular}{|c|c|c|c|c|c|}
\hline & $\begin{array}{c}\text { Total } \\
\text { number of } \\
\text { patients }\end{array}$ & $\begin{array}{l}\text { Patients on } \\
\text { topiramate } \\
(\%)\end{array}$ & $\begin{array}{l}\text { Odds } \\
\text { ratio* }\end{array}$ & $\begin{array}{l}95 \% \\
\text { Confidence } \\
\text { Interval }\end{array}$ & $p$ value \\
\hline \multicolumn{6}{|l|}{ Diagnostic category } \\
\hline Migraine & 3753 & $1832(48.8)$ & 1.91 & $(1.66,2.20)$ & $<0.0001$ \\
\hline Tension & 338 & $103(30.47)$ & 0.51 & $(0.40,0.64)$ & $<0.0001$ \\
\hline Cluster & 269 & $100(37.17)$ & 0.70 & $(0.54,0.90)$ & NS \\
\hline Other primary and NOS & 869 & $397(45.68)$ & 1.02 & $(0.88,1.18)$ & NS \\
\hline Secondary (post-traumatic and & 1164 & $553(47.51)$ & 1.12 & $(0.98,1.28)$ & NS \\
\hline \multicolumn{6}{|l|}{ IIH excluded) } \\
\hline Post-traumatic & 181 & $78(43.09)$ & 0.91 & $(0.67,1.23)$ & NS \\
\hline IIH & 178 & $135(75.84)$ & 3.97 & $(2.80,5.62)$ & $<0.0001$ \\
\hline Cranial neuralgias & 161 & $56(34.78)$ & 0.63 & $(0.46,0.88)$ & 0.0066 \\
\hline \multicolumn{6}{|l|}{ Co-existing diagnosis } \\
\hline Diabetes & 78 & $38(48.72)$ & 1.15 & $(0.73,1.80)$ & NS \\
\hline Overweight and obesity & 158 & $115(72.78)$ & 3.34 & $(2.34,4.77)$ & $<0.0001$ \\
\hline Anxiety and panic & 1053 & $505(47.96)$ & 1.14 & $(1.00,1.31)$ & NS \\
\hline Bipolar & 186 & $113(60.75)$ & 1.91 & $(1.42,2.58)$ & $<0.0001$ \\
\hline Depression & 1554 & $830(53.41)$ & 1.61 & $(1.43,1.82)$ & $<0.0001$ \\
\hline Seizures & 76 & $46(60.53)$ & 1.87 & $(1.17,2.97)$ & 0.0084 \\
\hline Fibromyalgia & 115 & $70(60.87)$ & 1.90 & $(1.30,2.78)$ & 0.0009 \\
\hline
\end{tabular}


*Odds ratios were calculated using simple logistic regression, and $p$ values are the associated $p$ values from the Wald Chi square tests associated with those univariate models.

$\mathrm{IIH}=$ idiopathic intracranial hypertension; NOS = not otherwise specified. 
Table 3 Total persistence on topiramate during study period by year and migraine status

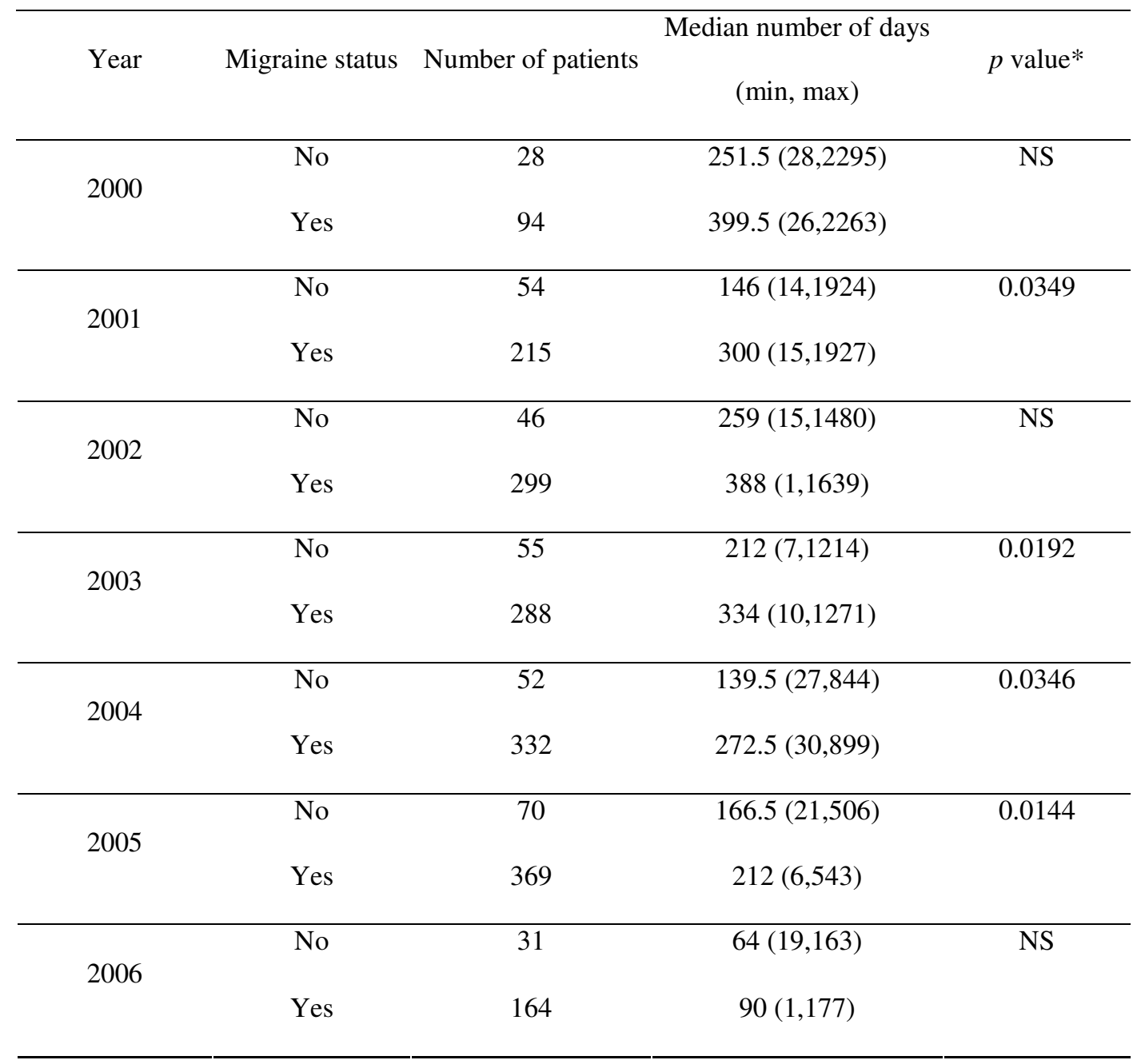

$* p$ values derived by the Wilcoxon test. 
Figure 1. Distribution of patients taking topiramate by maintenance and maximum daily dose. Maintenance dose is defined as the dose for the longest period of time during the study. All patients were included regardless of length of treatment. A total of 2192 patient records were evaluated.

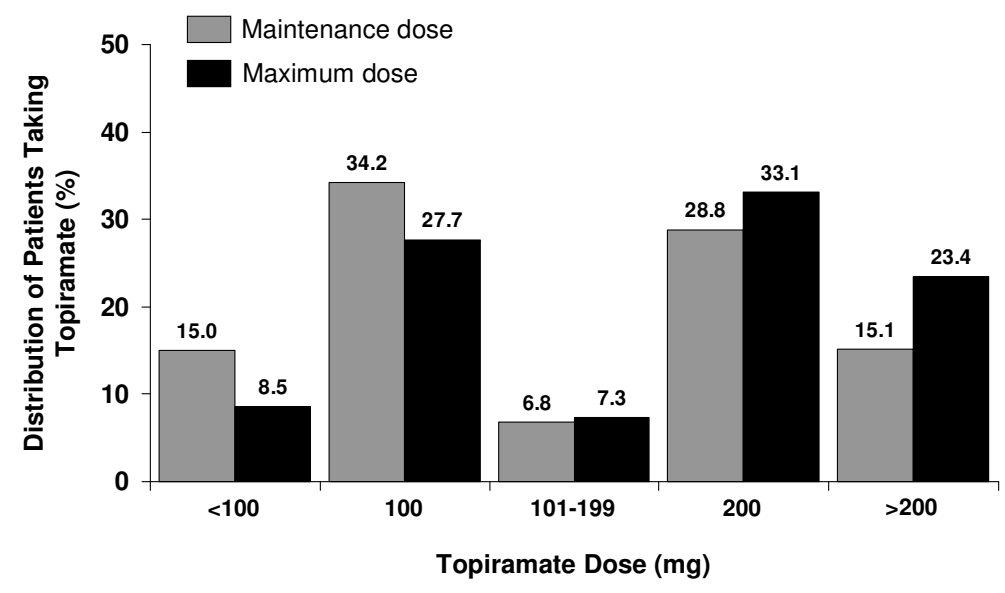


Figure 2. Persistence on topiramate based on the period patients took topiramate or projection from the number of pills. Total number of patient records evaluated: study period, $n=2097$; number of pills, $n=2098$.

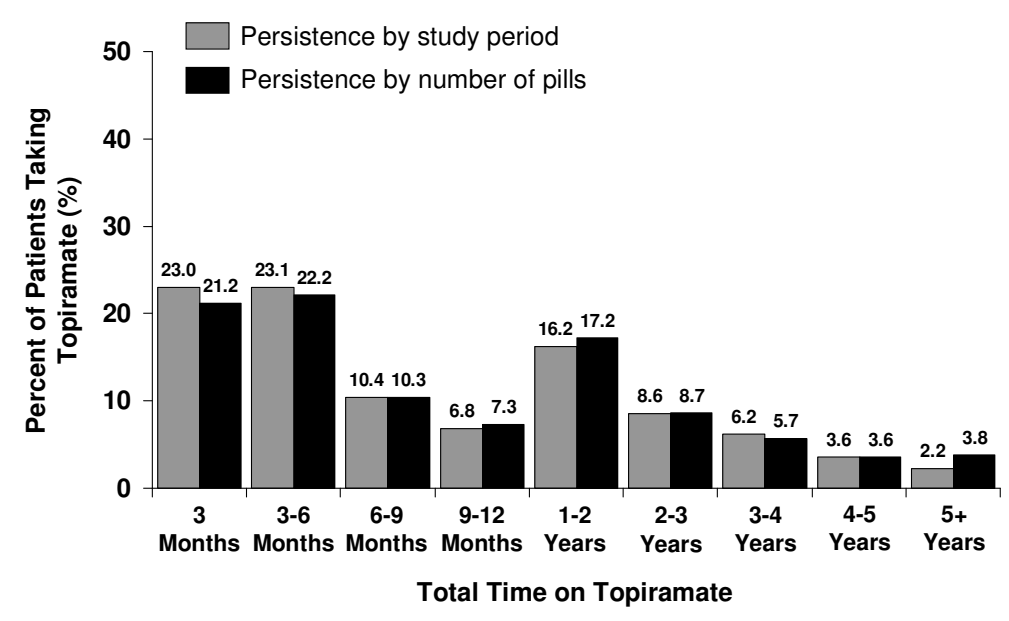

